\title{
The histone methyltransferase DOT1L is required for proper DNA damage response, DNA repair, and modulates chemotherapy responsiveness
}

Vijayalakshmi Kari ${ }^{1 \dagger}$, Sanjay Kumar Raul ${ }^{1,2 \dagger}$, Jana Maria Henck ${ }^{1}$ Julia Kitz ${ }^{3}$, Frank Kramer ${ }^{4,5}$, Robyn Laura Kosinsky ${ }^{1}$, Nadine Übelmesser ${ }^{6}$, Wael Yassin Mansour ${ }^{7,8}$, Jessica Eggert ${ }^{1}$, Melanie Spitzner ${ }^{1}$, Zeynab Najafova ${ }^{1}$, Holger Bastians ${ }^{6}$, Marian Grade', Jochen Gaedcke ${ }^{1}$, Florian Wegwitz ${ }^{1}$ and Steven A. Johnsen ${ }^{1 *}$ (D)

\begin{abstract}
Background: Disruptor of telomeric silencing 1-like (DOT1L) is a non-SET domain containing methyltransferase known to catalyze mono-, di-, and tri-methylation of histone 3 on lysine 79 (H3K79me). DOT1L-mediated H3K79me has been implicated in chromatin-associated functions including gene transcription, heterochromatin formation, and DNA repair. Recent studies have uncovered a role for DOT1L in the initiation and progression of leukemia and other solid tumors. The development and availability of small molecule inhibitors of DOT1L may provide new and unique therapeutic options for certain types or subgroups of cancer.

Methods: In this study, we examined the role of DOT1L in DNA double-strand break (DSB) response and repair by depleting DOT1L using siRNA or inhibiting its methyltransferase activity using small molecule inhibitors in colorectal cancer cells. Cells were treated with different agents to induce DNA damage in DOT1L-depleted or -inhibited cells and analyzed for DNA repair efficiency and survival. Further, rectal cancer patient samples were analyzed for H3K79me3 levels in order to determine whether it may serve as a potential marker for personalized therapy.

Results: Our results indicate that DOT1L is required for a proper DNA damage response following DNA double-strand breaks by regulating the phosphorylation of the variant histone $\mathrm{H} 2 \mathrm{AX}(\mathrm{\gamma H} 2 \mathrm{AX})$ and repair via homologous recombination (HR). Importantly, we show that small molecule inhibitors of DOT1L combined with chemotherapeutic agents that are used to treat colorectal cancers show additive effects. Furthermore, examination of H3K79me3 levels in rectal cancer patients demonstrates that lower levels correlate with a poorer prognosis.
\end{abstract}

Conclusions: In this study, we conclude that DOT1L plays an important role in an early DNA damage response and repair of DNA double-strand breaks via the HR pathway. Moreover, DOT1L inhibition leads to increased sensitivity to chemotherapeutic agents and PARP inhibition, which further highlights its potential clinical utility. Our results further suggest that H3K79me3 can be useful as a predictive and or prognostic marker for rectal cancer patients.

Keywords: DOT1L, H3K79me, YH2AX, DNA damage, DNA double-strand breaks, Homologous recombination, PARP inhibition, FOLFIRI, Colorectal cancer

\footnotetext{
* Correspondence: steven.johnsen@med.uni-goettingen.de

†Vijayalakshmi Kari and Sanjay Kumar Raul contributed equally to this work.

${ }^{1}$ Department of General, Visceral and Pediatric Surgery, University Medical

Center Göttingen, 37075 Göttingen, Germany

Full list of author information is available at the end of the article
}

(c) The Author(s). 2019 Open Access This article is distributed under the terms of the Creative Commons Attribution 4.0 International License (http://creativecommons.org/licenses/by/4.0/), which permits unrestricted use, distribution, and

reproduction in any medium, provided you give appropriate credit to the original author(s) and the source, provide a link to the Creative Commons license, and indicate if changes were made. The Creative Commons Public Domain Dedication waiver (http://creativecommons.org/publicdomain/zero/1.0/) applies to the data made available in this article, unless otherwise stated. 


\section{Background}

Colorectal cancer (CRC) is the third leading cause of cancer-related deaths worldwide [1]. The sequential accumulation of genetic mutations and epigenetic changes transforms the normal epithelia to adenoma and aggressive cancer [2]. Genomic instability is a hallmark of CRC, which is classified in major subtypes based on specific molecular signatures [3, 4]. Although recent advances in the treatment of CRC have increased patient survival, therapeutic resistance and relapse remain major challenges in treating CRC patients due to tumor heterogeneity and the development of resistance mechanisms [5].

DNA double-strand breaks (DSB) are the most lethal form of DNA damage, and unrepaired DSBs lead to chromosomal abnormalities and can result in a variety of pathological conditions including cancer [6]. In eukaryotes, the recognition and repair of DSBs is associated with post-translational histone modifications (PTMs) and requires chromatin remodeling and repair factors to enable proper recognition and repair of breaks and maintain genomic integrity [7]. One such modification is methylation of H3 at lysine 79 (H3K79me), mediated by the non-SET domain containing methyltransferase DOT1L, which catalyzes the mono-, di-, and tri-methylation of H3K79 $[8,9]$. Several studies have demonstrated a role of Dot1 or its mammalian homolog DOT1L in mediating H3K79me and controlling genomic processes including gene transcription, splicing, telomeric silencing, and DNA damage repair $[8,10-14]$. Further studies have implicated a role in heterochromatin maintenance and development [15]. Moreover, DOT1L interacts with the $M L L$ translocation protein complex, thereby leading to aberrant methylation of target genes, and is associated with tumorigenesis and poor outcome [16-18]. Recently developed small molecule inhibitors of DOT1L are currently being tested in the treatment of MLL-rearranged leukemia [19-21]. We previously identified the DOT1L gene as 1 of 11 genes whose increased methylation is associated with better disease outcome in rectal cancer patients [22]. Though previous studies have suggested a role of DOT1L in DNA repair and transcription recovery after DNA damage, its role in DSB repair and the potential utility of DOT1L inhibitors in combination with standard of care therapies of CRC remain largely unknown.

In this study, we demonstrate the importance of DOT1L-mediated H3K79me3 in the early DNA damage response and the repair of DNA DSBs. Depletion or inhibition of DOT1L methyltransferase activity leads to an impaired DNA damage response indicated by decreased $\gamma \mathrm{H} 2 \mathrm{AX}$ levels, but increased phosphorylation of KAP1. Importantly, the loss of DOT1L function leads to defective HR-mediated DSB repair without affecting NHEJ. Importantly, loss of DOT1L or inhibition of its methyltransferase activity increased sensitivity to irradiation and chemotherapeutic agents used in the treatment of CRC patients. Consistent with the finding that defects in HR-mediated DSB repair lead to sensitivity toward poly (adenosine diphosphate [ADP]) ribose polymerase (PARP) inhibitors [23, 24], inhibition of DOT1L increased sensitivity to PARP inhibitors, further confirming its role in HR-mediated repair. Finally, by examining a cohort of rectal cancer patient samples, we provide the first evidence that patients with low H3K79me3 display a tendency toward overall poorer survival, indicating that this subgroup of patients with decreased H3K79me3 levels may benefit from treatment with PARP inhibitors.

\section{Results}

DOT1L is required for proper DNA damage response

Phosphorylation of H2AX at serine 139 ( $\gamma \mathrm{H} 2 \mathrm{AX})$ by specific DNA damage response-associated members of the phosphatidylinositol-3-kinase family is an early marker of DNA damage induction. In order to examine a potential role of DOT1L in the DNA damage response to DNA double-strand breaks (DSB), initially DOT1L was efficiently depleted in U2OS osteosarcoma cells, a cell line widely used to study DNA repair mechanisms, and DSBs were induced by the radiomimetic drug neocarzinostatin (NCS). Western blot analysis with total protein lysates for $\gamma \mathrm{H} 2 \mathrm{AX}$ demonstrated increased $\gamma \mathrm{H} 2 \mathrm{AX}$ within $15 \mathrm{~min}$ of NCS treatment which decreased to basal levels by $6 \mathrm{~h}$, consistent with a near complete repair of DSBs. Interestingly, DOT1L-depleted cells showed only a moderate increase in the levels of $\gamma \mathrm{H} 2 \mathrm{AX} 15 \mathrm{~min}$ after DSB induction, suggesting that DOT1L depletion may compromise the early DNA damage response (Fig. 1a). Moreover, no further increase in $\gamma \mathrm{H} 2 \mathrm{AX}$ was observed at any of the time points analyzed. Given the fact that DOT1L methylates H3K79, we examined global H3K79me3 levels in both control cells and following DSB induction. In contrast to $\gamma \mathrm{H} 2 \mathrm{AX}, \mathrm{H} 3 \mathrm{~K} 79 \mathrm{me} 3$ levels were only moderately increased following DSB induction with significantly slower kinetics (Fig. 1a). As expected, H3K79me3 levels were significantly decreased in DOT1L-depleted cells (Fig. 1a). Moreover, we also observed increased phosphorylation of KAP1 at serine 824 (pKAP1) in DOT1L-depleted cells compared to control cells 15 min after DSB induction (Fig. 1a). Immunofluorescence studies using U2OS cells further confirmed that $\gamma \mathrm{H} 2 \mathrm{AX}$ induction is compromised, while pKAP1 levels were elevated in DOT1L-depleted cells (Fig. 1b, Additional file 1: Figure S1c). The quantification of $\gamma \mathrm{H} 2 \mathrm{AX}$ and pKAP1 levels from control and DOT1L-depleted cells confirm that the overall intensity of $\gamma \mathrm{H} 2 \mathrm{AX}$ is decreased, while pKAP1 levels were elevated in DOT1L-depleted cells compared to control cells $15 \mathrm{~min}$ 
a

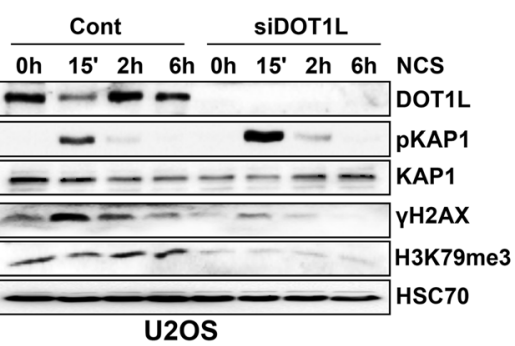

b

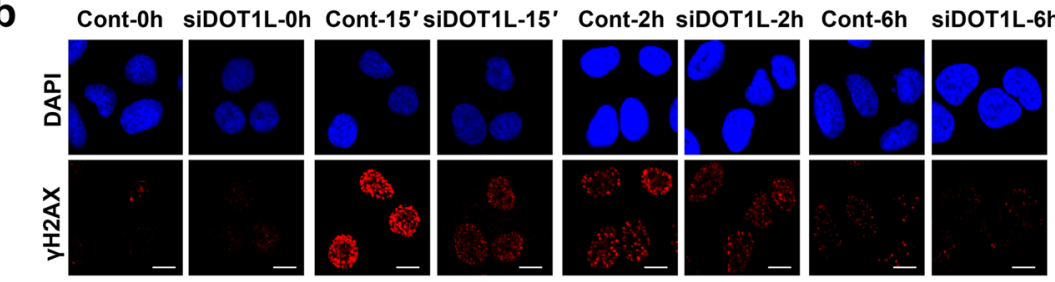

C
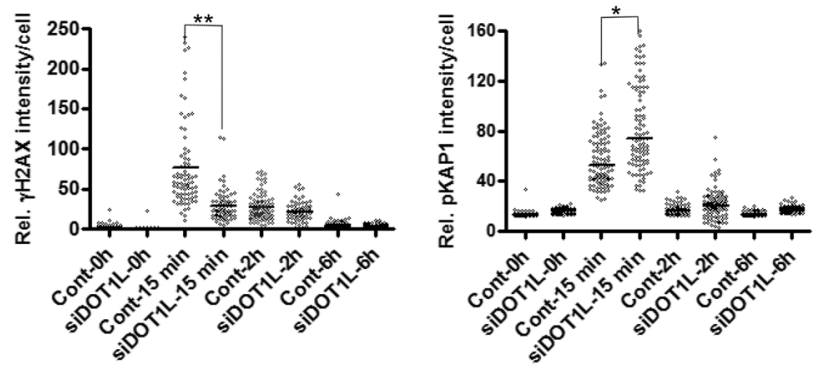

d

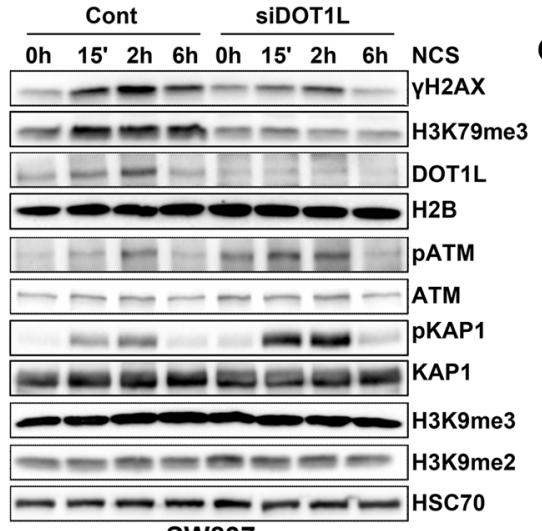

NCS
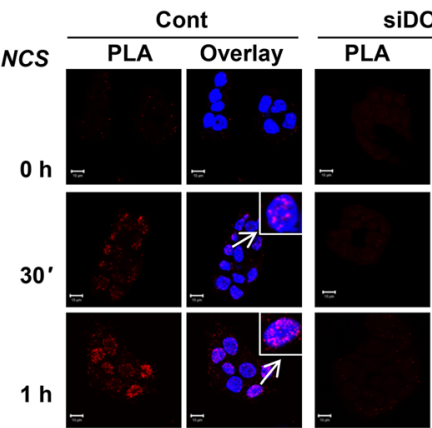

Overlay

$\mathbf{O h}$

$30^{\prime}$
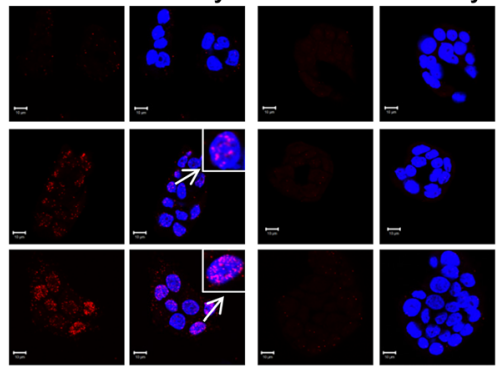

SW837

Fig. 1 DOT1L is required for the proper DNA damage response. a U2OS cells were transfected either mock (Control—Cont) or with siRNA (smart pool of 4 siRNA - SP) for DOT1L. After 48 h of transfection, cells were treated with neocarzinostatin (NCS; $100 \mathrm{ng} / \mathrm{ml}$ ) to induce DNA DSBs for the indicated time points. Total protein lysates were analyzed by Western blot for the indicated proteins. $\mathbf{b}$ Similar to $\mathbf{a}$, U2OS cells were transfected with siRNA and after $48 \mathrm{~h}$ of transfection, cells were treated with NCS for the indicated time points and processed for immunofluorescence analysis for $\gamma \mathrm{H} 2 \mathrm{AX}$ in red and nuclei were stained with DAPI in blue (Scale bar-10 $\mu \mathrm{M})$. c Quantification of $\mathrm{YH} 2 \mathrm{AX}$ and pKAP1 levels in mock or DOT1L siRNA-transfected U2OS cells treated with NCS for the indicated times from b. More than 50 cells were measured for each condition and represented as relative intensity in each cell, mean values are represented in the plot $n=3, \pm$ SD, $p$ values $\left({ }^{*} 0.005\right.$ and $\left.{ }^{*} 0.01\right)$. d SW837 cells were either mock (Cont) transfected or with DOT1L siRNA, after $72 \mathrm{~h}$ cells were treated with NCS $(100 \mathrm{ng} / \mathrm{ml})$ to induce DSBs and total protein lysates were immunoblotted with the indicated antibodies. e Proximity ligation assay (PLA) for $\gamma H 2 A X$ and H3K79me3 in SW837 cells transfected with either mock (Cont) or DOT1L siRNA for $72 \mathrm{~h}$ prior to treatment with NCS (100 ng/ml) for the indicated time points before processing for PLA. Red dots indicate regions of close proximity between $\mathrm{YH} 2 \mathrm{AX}$ and H3K79me3. Nuclei were stained with DAPI (Scale bar-10 $\mu \mathrm{M}$ )

after DNA DSB induction (Fig. 1c) and the effects were consistent over time. Similar results were observed in the rectal cancer cell line SW837, where the depletion of DOT1L (Additional file 1: Figure S1a, b) also led to decreased induction of $\gamma \mathrm{H} 2 \mathrm{AX} 15$ min after DSB induction (Fig. 1d), while pKAP1 levels were elevated compared to control cells (Fig. 1d, Additional file 1: Figure S1d). We also observed some variation in DOT1L levels following 
DSB induction (Fig. 1a, d); however, these effects were highly variable between cell lines and experiments. Next, we analyzed the activation of ataxia telangiectasia mutated kinase (ATM), an upstream kinase for H2AX, which is auto-phosphorylated at serine 1981 upon DSB induction. Interestingly, DOT1L-depleted cells displayed slightly elevated levels of phosphorylated ATM compared to control cells upon DSB induction, suggesting that the observed decrease in $\gamma \mathrm{H} 2 \mathrm{AX}$ levels following DOT1L depletion was not due to defective upstream kinase activity (Fig. 1d, Additional file 1: Figure S1e). This further suggests that DOT1L or DOT1L-mediated H3K79 methylation controls the early DNA damage response pathway.

Since phosphorylation of KAP1 is associated with DNA repair in heterochromatic regions, we also examined whether DOT1L depletion may elicit global changes in heterochromatin markers. However, no changes in H3K9me2, H3K9me3, or HP1 levels were observed in DOT1L-depleted cells (Fig. 1d, Additional file 1: Figure S1g), suggesting that the observed effects on KAP1 phosphorylation are not simply due to a global re-partitioning of euchromatin into heterochromatin. Since earlier studies indicated that DOT1L or DOT1L-mediated H3K79me may play an important role in the early DNA damage response, we used the proximity ligation assay (PLA) to examine whether H3K79me3 and $\gamma \mathrm{H} 2 \mathrm{AX}$ co-localize upon DSB induction. Indeed, while global H3K79me3 levels were unchanged following DSB induction, $\gamma \mathrm{H} 2 \mathrm{AX}$ and H3K79me3 were co-localized within 30 min following DSB induction and a further increase was observed at $1 \mathrm{~h}$ after DSB induction. The specificity of the assay was confirmed by DOT1L depletion, which led to decreased H3K79me3 levels and a loss of PLA signal (Fig. 1e). The co-localization of $\gamma \mathrm{H} 2 \mathrm{AX}$ and 53BP1 served as a positive control for the experiment (Additional file 1: Figure S1f, g).

\section{DOT1L is required for homologous recombination- mediated DSB repair}

Based on our results indicating that DOT1L is required for the early DNA damage response, we further investigated a role for DOT1L in DSB repair. Initially, control and DOT1L-depleted SW837 cells were irradiated with 6 Gy to induce DSBs and total protein lysates were analyzed by Western blot analysis. Similar to our results using NCS, DOT1L depletion resulted in decreased induction of $\gamma \mathrm{H} 2 \mathrm{AX}$ compared to control cells (Fig. 2a). We further analyzed the sensitivity of DOT1L-depleted cells toward irradiation (Fig. 2b). Colony formation assays confirmed that DOT1L-depleted cells were more sensitive to irradiation compared to the control cells, suggesting that DOT1L may be required for DSB repair (Fig. 2c). Since DSBs are repaired by two major pathways, namely by the error prone non-homologous end joining (NHEJ) and error-free homologous recombination (HR) pathways [25], we next sought to determine whether DOT1L preferentially promotes repair of DSBs by one or both of these pathways. Therefore, DOT1L was depleted in HeLa cells harboring single copies of a plasmid-based GFP-reporter specific for NHEJ- or HR-mediated DNA DSB repair (pEJ and pGC, respectively). This system allows for a precise measurement of the efficiency of NHEJ and HR based on the number of $\mathrm{GFP}^{+}$cells measured by flow cytometry. Interestingly, depletion of DOT1L using two different siRNAs led to decreased HR-mediated repair without impairing NHEJ-mediated repair (Fig. 2d). Importantly, these findings could be confirmed in HCT116 cells harboring the stably integrated HR substrate (Fig. 2e and Additional file 2: Figure S2a). To further validate the role of DOT1L in HR-mediated DSB repair, we performed Western blot analysis of chromatin fractions from control and DOT1L-depleted SW837 cells after DSB induction. Repair of DSBs via HR is particularly dependent upon the resection of one strand of DNA at the break sites. This process is controlled by many proteins where, in particular, CtBP-interacting protein (CtIP) is recruited at an early stage of the end resection process [26]. Our results show that while CtIP is recruited to chromatin in control cells following DSB induction; this recruitment is severely compromised in DOT1L-depleted cells, indicating that DNA end resection is defective in the absence of DOT1L (Fig. 2f). End resection generates a stretch of 3' single-stranded DNA (ssDNA) that is coated with the single-stranded DNA (ssDNA) binding proteins RPA1 and RAD51. Since we observed decreased CtIP recruitment in DOT1L-depleted cells, we next examined the binding of RPA1 and RAD51 proteins to the chromatin as an indicator of end resection processing and ssDNA generation. Consistent with our findings that CtIP recruitment was impaired following DOT1L depletion, we also observed decreased recruitment of RPA1 and RAD51 to DNA in DOT1L-depleted cells (Fig. 2f). Consistently, RAD51 phosphorylation was also decreased in DOT1L-depleted cells (Additional file 2: Figure S2b) and the effects were not due to changes in cell cycle regulation upon DOT1L depletion (Additional file 2: Figure S2c). Thus, together, these findings indicate that DOT1L is required specifically in the end resection step of HR-mediated DSB repair.

\section{Inhibition of DOT1L leads to increased sensitivity to DNA damaging agents}

We next sought to determine whether pharmacological inhibition of DOT1L methyltransferase activity, using the recently developed small molecular inhibitor EPZ5676 (EPZ; also called pinometostat) [19, 27], which was tested in phase I clinical trials for adult and pediatric MLL-translocated leukemia (NCT01684150 and 
a

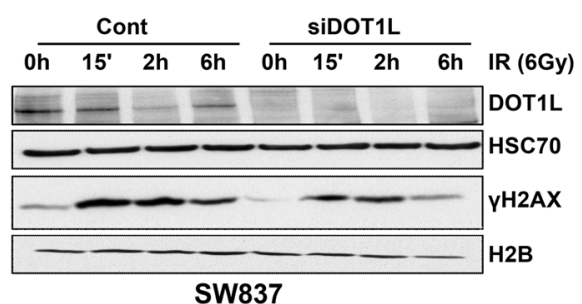

b

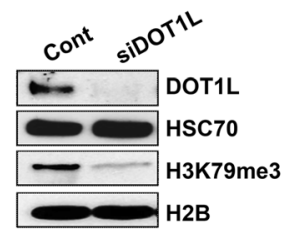

C

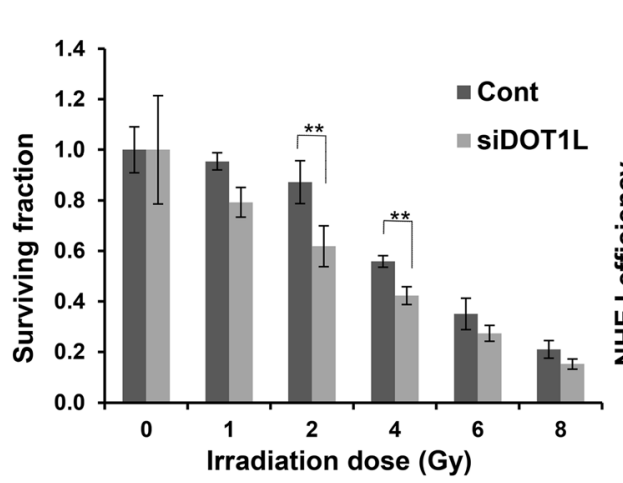

e

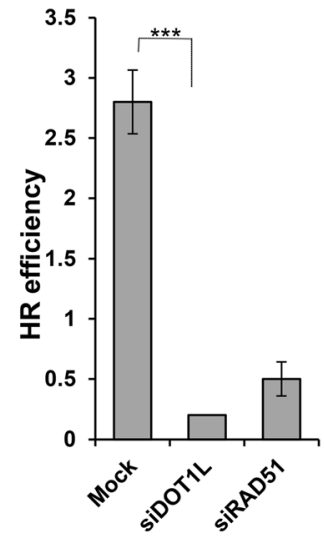

d

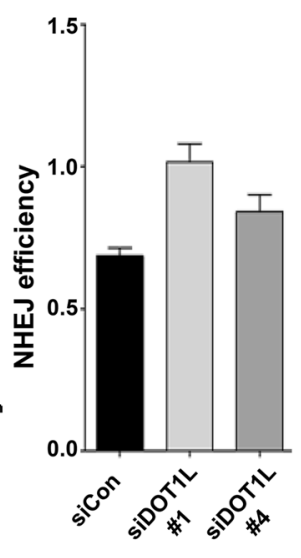

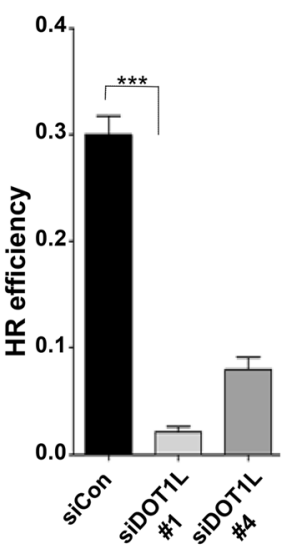

siDOT1L

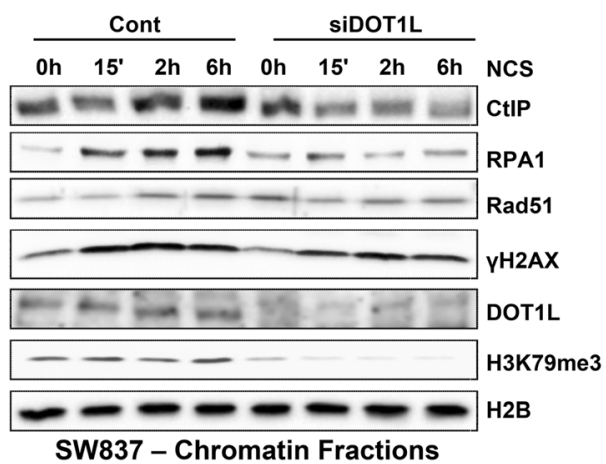

SW837 - Chromatin Fractions

Fig. 2 DOT1L is required for homologous recombination-mediated DNA DSB repair. a SW837 cells were transfected either mock or with DOT1L siRNA (SmartPool) for $48 \mathrm{~h}$, treated with ionizing radiation ( $6 \mathrm{~Gy}$ ), and total protein lysates were harvested at the indicated time points and immunoblotted with the indicated antibodies. $\mathrm{H} 2 \mathrm{~B}$ and $\mathrm{HSC70}$ were used as loading controls. b DOT1L knockdown efficiency in siRNAtransfected SW837 cells (parallel to a) was verified in whole cell lysates with DOT1L and H3K79me3 antibodies. c Colony formation assays were performed with control and DOT1L-depleted SW837 cells from b with the indicated doses of irradiation (Gy) and data represent the mean values from the surviving fraction (SF), $n=3, \pm S D, p$-value (0.005-4 Gy). $\mathbf{d}$ HeLa cells harboring single copies of an NHEJ repair substrate (pEJ) or an HR repair substrate (pGC) were transfected with either mock or DOT1L siRNA \#1 or \#4 and a DSB was induced by transfecting cells with an I-Scel-expression vector (pCMV-I-Scel-3xNLS). After $48 \mathrm{~h}$, the percentage of GFP-positive cells was measured using flow cytometry analysis as an indication for HR and NHEJ efficiency. e HCT1 16 cells with an integrated GFP reporter system for HR efficiency were transfected with the indicated siRNAs and GFP-positive cells were measured using flow cytometry analysis. $\mathbf{f}$ SW837 cells were transfected as in b and $72 \mathrm{~h}$ after transfection treated with NCS (100 ng/ml) for the indicated time points. Chromatin fractions were harvested and subjected to Western blot analysis for the indicated proteins

NCT02141828, respectively) [28], also impairs the DNA damage response and repair. Thus, DOT1L activity was inhibited by EPZ treatment of SW837 cells for either $3 \mathrm{~h}$ (acute inhibition) or $72 \mathrm{~h}$ (prolonged inhibition) followed by treatment with NCS to induce DSBs. Consistent with siRNA-mediated DOT1L depletion, Western blot analysis of total protein lysates revealed that both acute and prolonged DOT1L inhibition result in decreased accumulation of $\gamma \mathrm{H} 2 \mathrm{AX}$ (Additional file 3: Figure S3a). Notably, a decrease in global H3K79me3 was only observed after prolonged (72 h) EPZ treatment, but relatively unaffected within $3 \mathrm{~h}$ (Additional file 3: Figure S3a, Fig. 3a), 
a

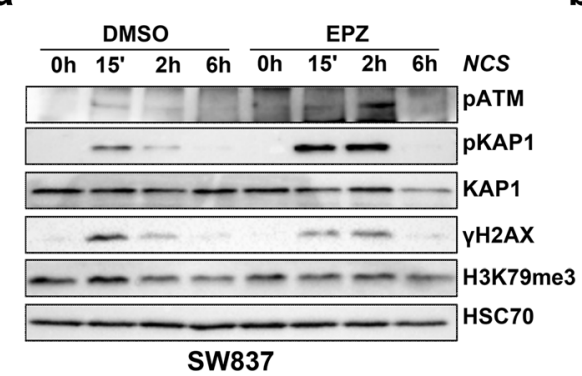

C

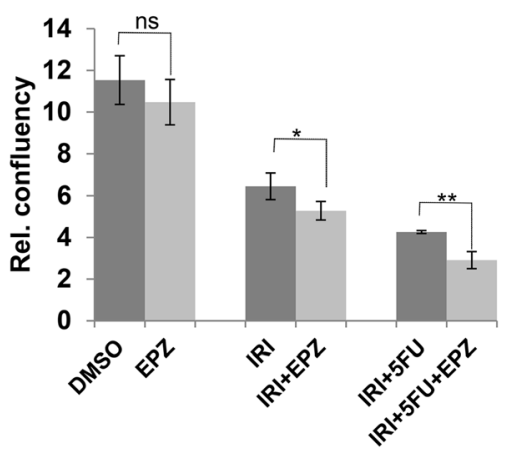

b

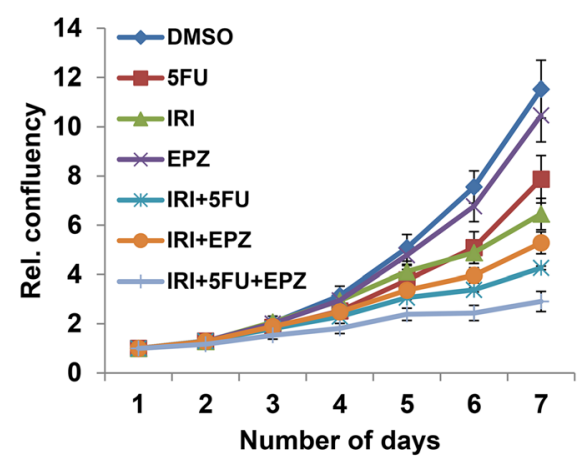

d

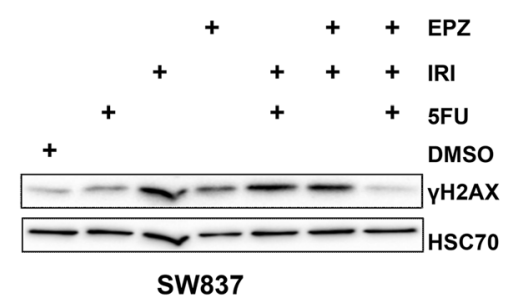

Fig. 3 Inhibition of DOT1L leads to increased sensitivity to DNA damaging agents. a SW837 cells were pretreated with either with DMSO or the DOT1L inhibitor EPZ5676 (1 $\mu \mathrm{M})$ for $3 \mathrm{~h}$ followed by treatment with NCS $(100 \mathrm{ng} / \mathrm{ml})$ for the indicated time points. Whole cell lysates were immunoblotted and detected with the indicated antibodies. b Cell proliferation assay with SW837 cells treated either with 5-fluorouracil (5-FU; $5 \mu \mathrm{M})$, Irinotecan (IRI; $1 \mu \mathrm{M})$, EPZ5676 (1 $\mu \mathrm{M})$ individually or in combination as indicated. Cell confluency was measured every $24 \mathrm{~h}$ using a Celigo cytometer. $\mathbf{c}$ Bar graph representation of cell proliferation analysis from $\mathbf{b}$ at day 7 . Data are represented as mean values $n=3, \pm \mathrm{SD}$, and $p$ values $\left(\mathrm{ns},{ }^{*} 0.01,{ }^{* *} 0.003\right)$. $\mathbf{d}$ Western blot analysis from SW837 cells treated as in $\mathbf{b}$ for $48 \mathrm{~h}$ with the indicated compounds

suggesting that DSB-induced DOT1L activity at sites of DNA damage, rather than pre-existing H3K79me3, may be important for HR-mediated DNA repair. Similar results were observed using another chemically distinct DOT1L inhibitor SGC094a, where treatment for 3 or $72 \mathrm{~h}$ similarly led to decreased $\gamma \mathrm{H} 2 \mathrm{AX}$ levels following NCS treatment (Additional file 3: Figure S3b). Moreover, phospho-ATM and phospho-KAP1 levels were also increased following DOT1L inhibition for $3 \mathrm{~h}$ (Fig. 3a). Together, these findings demonstrate that DOT1L methyltransferase activity is specifically required for a proper DNA damage response.

Given the potential clinical application of DOT1L inhibitors in cancer treatment, we next tested the effects of combinatorial treatment with standard of care therapies for colorectal cancer, where 5-fluorouracil (5-FU), a nucleoside analogue, is frequently combined with the clinically utilized topoisomerase I inhibitor irinotecan (IRI) in a combination referred to as FOLFIRI $[29,30]$. Thus, we tested how treatment with the clinical DOT1L inhibitor EPZ5676 would impact the effects of the individual and combined treatments with IRI and 5-FU on cell proliferation and survival. As expected, individual treatments with IRI or 5-FU alone decreased the proliferation of SW837 cells and combination of the two treatments further decreased cell proliferation. In contrast, EPZ treatment alone had very little effect on cell proliferation, but enhanced the anti-proliferative effects of 5-FU, IRI, and combined treatment (Fig. 3b, c). The observed effects are likely due to an impaired DNA damage response since global $\gamma \mathrm{H} 2 \mathrm{AX}$ levels were increased by IRI, 5-FU, and combined IRI/5-FU $48 \mathrm{~h}$ after treatment, while the combination of IRI/5-FU and EPZ resulted in decreased $\gamma \mathrm{H} 2 \mathrm{AX}$ levels compared to IRI, 5-FU, or IRI/5-FU (Fig. 3d).

\section{Inhibition of DOT1L leads to increased sensitivity to PARP inhibition}

A unique and therapeutically useful feature of tumor cells with HR deficiencies (e.g., BRCA1 mutations) is an increased sensitivity to PARP inhibitors [23, 31]. Since we specifically observed defective HR-mediated DSB repair in DOT1L-depleted cells and following inhibition of DOT1L methyltransferase activity, we hypothesized that loss of DOT1L activity may lead to sensitivity to PARP inhibitors. In order to test this, we treated SW837 cells 
with a DOT1L inhibitor (EPZ), IRI, the PARP inhibitor Veliparib (VEL), or combinations thereof. Consistent with our hypothesis, inhibition of DOT1L methyltransferase activity led to increased sensitivity toward IRI or VEL, with the combination of all three agents having the greatest effect on SW837 rectal carcinoma cells (Fig. 4a, b). Similar results were observed with U2OS cells, where the combination of EPZ, IRI, and VEL also decreased the proliferation of cells more than the individual treatments (Fig. 4d, e). Consistent with the effects observed with NCS, irradiation, and IRI/5-FU treatments, activation of the DNA damage response (e.g., $\gamma \mathrm{H} 2 \mathrm{AX}$ levels) was decreased following the combination treatment in both cell lines (Fig. 4c, f).

\section{Patients with low H3K79me3 levels show poorer overall survival}

Defects in HR-mediated DNA repair are frequently associated with poorer patient survival. Thus, we hypothesized that DOT1L-mediated H3K79me3 levels may correlate with patient outcome in colorectal cancer. In order to test this, we performed immunohistochemistry analysis for H3K79me3 on tumor microarrays (TMAs) derived from treatment naïve rectal cancer patients $(n=156)$. Interestingly, while normal mucosa consistently displayed low levels of H3K79me3, tumors displayed varying levels of H3K79me3, with many displaying heterogeneous staining within the tumor (Fig. 5a). Notably, low or heterogeneous H3K79me3 levels were associated with an overall poorer prognosis compared to patients with tumors displaying higher levels of H3K79me3 showing a better overall survival (Fig. 5b). Consistent with these findings, analysis of DOT1L mRNA levels in rectal adenocarcinoma patient gene expression data from the TCGA research network (http://cancergenome.nih.gov/) revealed a similar trend with patients displaying higher DOT1L expression having a significantly better overall survival compared to patients with lower DOT1L expression levels (Fig. 5c).

\section{Discussion}

Proper DNA damage recognition and response are key steps necessary to repair DSBs in a timely manner and to prevent genomic instability and the development of cancer. Histone modifications and chromatin remodelers play an important role in repairing DNA damage, especially at DSBs $[6,7]$. In this study, we elucidated a function of the H3K79 histone methyltransferase DOT1L in controlling the DNA damage response and repair of DSBs.

Our results show that depletion of DOT1L impaired the DNA damage response as assessed by the accumulation of $\gamma \mathrm{H} 2 \mathrm{AX}$ at 15 min following DSB induction. This indicates that DOT1L plays an essential central role in the early DNA damage response, while the activation of the upstream kinase ATM (as assessed by autophosphorylation of Ser1981) was not inhibited by DOT1L depletion or inhibition. Interestingly, DOT1L depletion or inhibition resulted in increased accumulation of phosphorylated KAP1, a protein associated with DNA repair at heterochromatic regions, indicating that DOT1L likely also influences DSB repair in heterochromatin [32, 33]. Since we did not observe global changes in the heterochromatin markers $\mathrm{H} 3 \mathrm{~K} 9 \mathrm{me} 2 / 3$, this effect does not appear to be due to global changes in chromatin compartmentalization. Importantly, these effects could be observed after acute treatment $(3 \mathrm{~h})$ with the DOT1L methyltransferase inhibitor EPZ5676, which did not cause a global loss of H3K79me3, suggesting that DOT1L likely plays a direct methyltransferase-dependent function at DSBs, rather than affecting DSB repair via the transcription-associated marking of active chromatin regions. This finding is consistent with our proximityligation assays demonstrating a co-localization of H3K79me3 with $\gamma \mathrm{H} 2 \mathrm{AX}$ after DSB induction.

Consistent with a role of DOT1L in DNA repair processes, our results show that DOT1L-depleted cells are more sensitive to ionizing irradiation. Plasmid-based GFP-reporter assays suggest that this effect is likely due to impaired HR-, but not NHEJ-mediated DSB repair. This finding is supported by the observation that recruitment of CtIP, a protein critical in the DNA end resection process during HR-mediated repair, as well as the subsequent loading of the ssDNA binding proteins RPA1 and RAD51 to chromatin is impaired in the absence of DOT1L activity. Thus, DOT1L may cooperate with other chromatin remodeling factors such as CHD1 to specifically open chromatin to promote the DNA end resection process [34].

The DOT1L inhibitor Pinometostat (EPZ5767) used in this study recently completed a phase I clinical trial for adult leukemia (NCT01684150) and was shown to have modest activity as a single agent, but was generally safe and, therefore, potentially suitable for combinatory approaches with other chemotherapeutic agents [28]. Indeed, our study suggests that combining EPZ with standard of care treatment for colorectal cancer (FOLFIRI) can significantly increase therapeutic efficacy. This effect is likely mechanistically linked to the central role of DOT1L in HR-mediated DNA repair. Consistently, in much the same way that the combination of EPZ, VEL, and IRI significantly decreased cell survival compared to the individual treatments, we also showed that inhibition of DOT1L activity also increased sensitivity towards PARP inhibitors.

Our previous work identified the DOT1L gene as a differentially methylated gene in rectal cancer patients whose methylation levels in pre-therapeutic rectal cancer biopsies was associated with a better outcome [22]. 
a

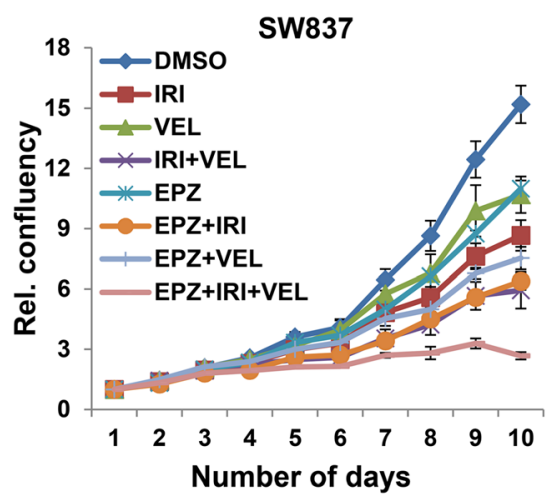

b

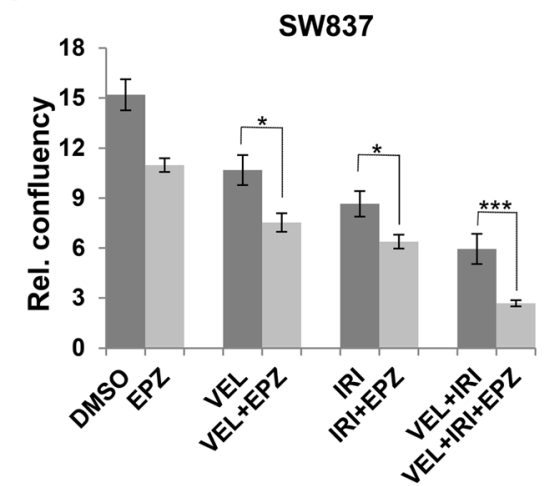

C

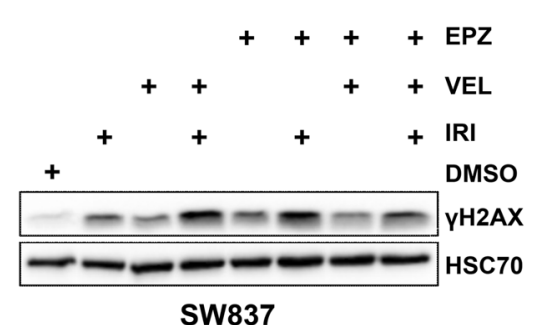

d

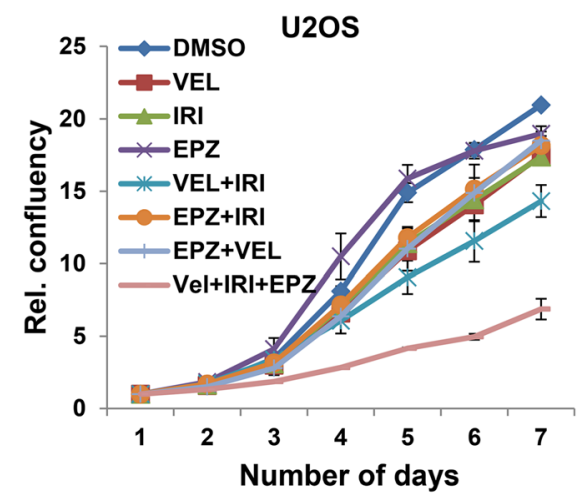

e

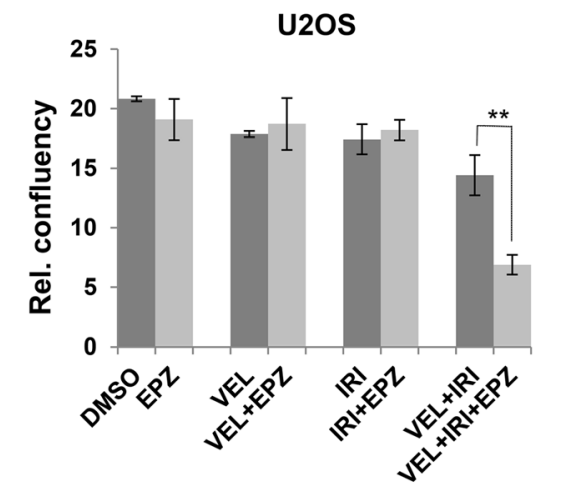

f

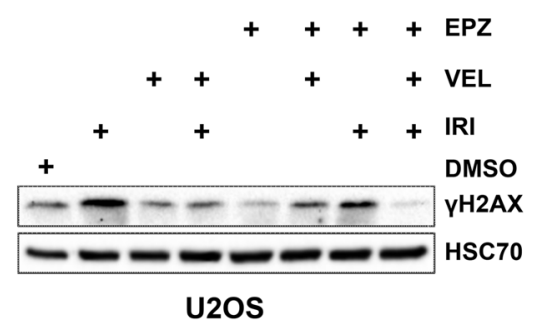

Fig. 4 Inhibition of DOT1L leads to increased sensitivity to PARP inhibition. a, d Cell proliferation of SW837 (a) and U2OS (d) cells treated either with IRI (1 $\mu \mathrm{M})$, EPZ (1 $\mu \mathrm{M})$, or Veliparib (VEL) individually or in combination and measured every $24 \mathrm{~h}$ using the Celigo cytometer. b, e Bar graph representation of cell proliferation of SW837 cells from $\mathbf{a}$ at day 10 and U2OS cells from $D$ at day 7. Data are represented as mean values $n=3, \pm$ SD, and $p$ value $\left(\mathbf{b}{ }^{*} 0.01,{ }^{*} 0.03,{ }^{* *} 0.0001 ; \mathbf{e}^{* *} 0.002\right)$. $\mathbf{c}$, f Western blot analysis from SW837 (c) and U2OS (f) cells treated as in (a, d) after 48 h of treatment. Total protein lysates were harvested and analyzed by Western blot using antibodies against the indicated proteins

Interestingly, in this study, no correlation between gene expression and DNA methylation could be observed, possibly implying that the expression of DOT1L following therapeutic challenge (rather than prior to treatment) may be important for enabling tumor cells to respond to therapeutic insult. In this study, we built upon these findings and examined whether rectal cancer patients may potentially be classified based on H3K79me3 levels or DOT1L mRNA levels. Indeed, when patient samples were classified into two major categories having either low or heterogeneous versus high H3K79me3 levels, we observed that low/heterogeneous H3K79me3 levels correlated with a poorer patient prognosis. Consistently, higher DOT1L mRNA levels also correlated with a better overall survival. Thus, H3K79me3 levels and/or DOT1L mRNA levels may potentially serve as prognostic markers, and potentially predict differential responsiveness to different standard of care treatments as well as sensitivity to PARP inhibitors.

\section{Conclusion}

In this study, we show that DOT1L is required for the proper DNA damage response following DSBs induction. Moreover, cells with decreased DOT1L activity show hypersensitivity to irradiation and display defects in 
a
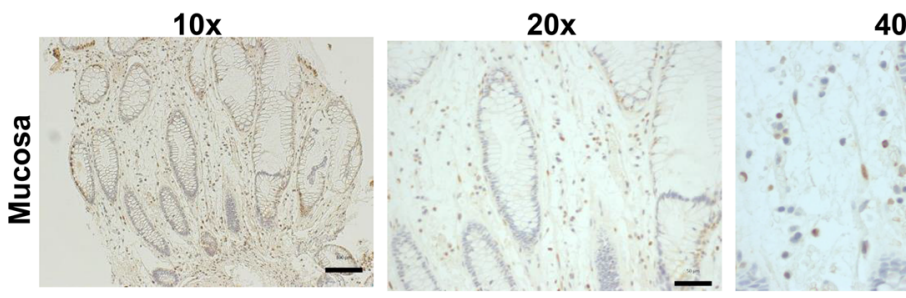

$40 x$
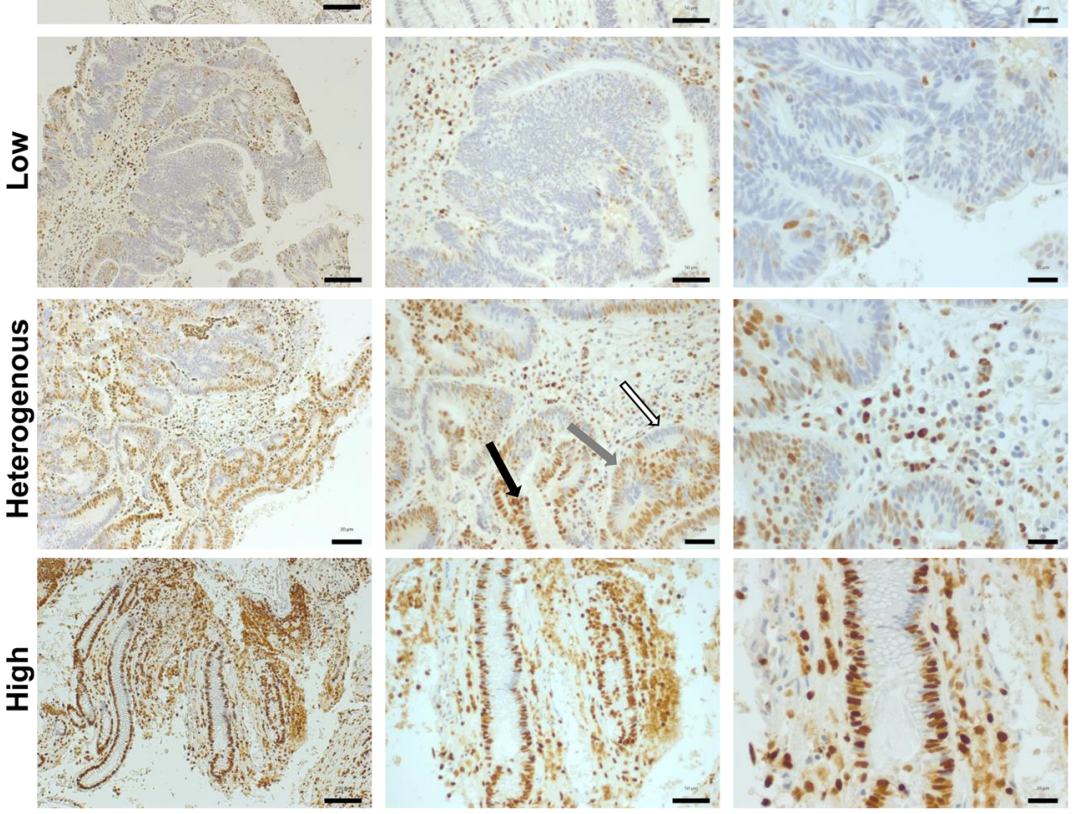

b

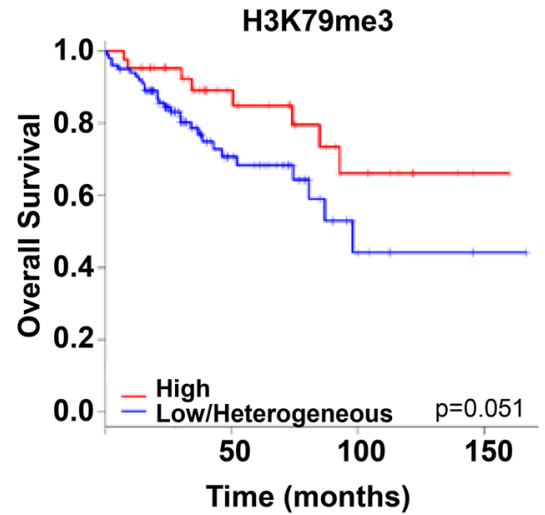

C

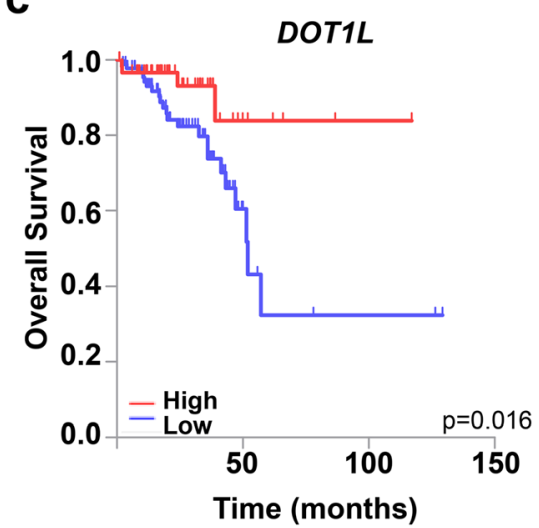

Fig. 5 Patients with low H3K79me3 or DOT1L mRNA levels show poorer overall survival. a, b In total, 156 rectal cancer patient biopsy samples from treatment naïve patients were analyzed by immunohistochemistry for H3K79me3. Based on the staining intensity, the nuclei were given the relative score of 0 to 3 , with 3 corresponding to highest staining intensity. Moreover, the overall percentage of the stained nuclei corresponding to each score was estimated for each analyzed sample. a Patient tissue samples were then classified based on the staining intensity and overall percentage of the stained nuclei as no staining, or low (5\% of the nuclei with low score), medium/heterogeneous (50\% of the nuclei stained heterogeneously), or high (100\% of the nuclei with high score) staining. Solid arrows indicate positive, gray arrows weak, and white arrows negative staining. b The Kaplan-Meier survival plot for groups of patients with tissue samples classified as "High" (red line) and "Low or Heterogeneous" (blue line; comprising patients with no, low or heterogeneous staining) ( $p$ value, 0.051). c Separation of rectal adenocarcinoma patients from The Cancer Genome Atlas into "high" ( $n=67)$ and "low" ( $n=92)$ DOT1L mRNA expression (greater than or less than 4.7 reads per kilobase per million, respectively) displayed in the Kaplan-Meier survival plot revealed better overall survival ( $p$ value, 0.018 ) in patients with higher levels of DOT1L expression

HR-mediated DNA repair. Consistently, small molecule inhibition of DOT1L methyltransferase increases sensitivity to chemotherapeutic agents such as 5-FU, irinotecan, and PARP inhibitor, suggesting its potential as a therapeutic target in rectal cancer. Future studies will be needed to further test the in vivo therapeutic 
efficacy of these findings and determine whether a combined inhibitor approach and/or H3K79me3-/ DOT1L-based patient stratification may serve to improve patient outcome.

\section{Methods}

\section{Cell culture and siRNA transfections}

U2OS, SW837, and HCT116 cells were cultured in Dulbecco's modified Eagle's medium (DMEM) (Invitrogen, DMEM/F12, and Roswell Park Memorial Institute medium (RPMI 1640)) respectively, supplemented with $10 \%$ fetal bovine serum (FBS) and $1 \mathrm{x}$ penicillin-streptomycin (Sigma, St. Louis, USA) at $37{ }^{\circ} \mathrm{C}$ under humidified condition with $5 \% \mathrm{CO}_{2}$. HeLa cells harboring $\mathrm{pEJ}$ or pGC substrates were grown in DMEM medium containing $800 \mu \mathrm{g} / \mathrm{ml} \mathrm{G} 418$ or $1 \mu \mathrm{g} / \mathrm{ml}$ puromycin, respectively. For transient depletion of DOT1L, siRNA transfections (sequences in Table 1) were performed using Lipofectamine RNAiMAX (Invitrogen, Waltham, USA) according to the manufacturer's instructions. Primer sequences for the verification of depletion of DOT1L mRNA levels are indicated in Table 2. For DOT1L transfections, we used SmartPool (Dharmacon) of four siRNAs (Table 1) or the indicated individual siRNAs. After $72 \mathrm{~h}$ of transfection, DNA double-strand breaks were either induced with neocarzinostatin (NCS, N9162, Sigma, $100 \mathrm{ng} / \mathrm{ml}$ ) or with ionizing radiation as indicated. To inhibit the methyltransferase activity of DOT1L, cells were treated with either EPZ5676 (S7062; Selleckchem) or with SGC094 (SML1107, Sigma) as indicated or in combination with 5-fluorouracil (5-FU, F6627; Sigma), Veliparib (ABT-888, S1004; Selleckchem), or irinotecan (I1406; Sigma) (Table 2).

\section{Immunofluorescence}

Cells were grown on sterile coverslips and transfected with the indicated siRNAs. After $72 \mathrm{~h}$ of transfection, the cells were treated with neocarzinostatin (NCS; N9162, Sigma-Aldrich) as indicated. Cells were fixed with $4 \%$ paraformaldehyde for $10 \mathrm{~min}$ at room temperature and then permeabilized with $0.1 \%$ Triton $\mathrm{X}-100$ in PBS for $5 \mathrm{~min}$. Cells were blocked with 3\% bovine serum albumin (BSA) in PBS and incubated

Table 1 List of siRNA used in this study

\begin{tabular}{ll}
\hline Gene & Sequence 5' to 3' \\
\hline siDOT1L-1 & CGAAGUGGAUGAAAUGGUA \\
siDOT1L-2 & CCGAGAAGCUCAACAACUA \\
siDOT1L-3 & GAAGCCGUCUCCCUCCAAA \\
SiDOT1L-4 & GAAACAAGCUCUAGAUCAU \\
Luciferase GL2 duplex & GCAGAAUCGUGUCCUCGAA \\
siGENOME non-targeting & D-001206-13-05 (Dharmacon) \\
siRNA pool \# 1 & \\
\hline
\end{tabular}

Table 2 RT-PCR primers used in this study

\begin{tabular}{lll}
\hline Name & Sequence & Source \\
\hline h DOT1L For & CCACCAACTGCAAACATCAC & This study \\
h DOT1L Rev & AGAGGAAATCGCCTCTCTCC & This study \\
HNRNPK For & ATCCGCCCCTGAACGCCCAT & {$[36]$} \\
HNRNPK Rev & ACATACCGCTCGGGGCCACT & {$[36]$} \\
\hline
\end{tabular}

overnight with primary antibody at $4{ }^{\circ} \mathrm{C}$ in $3 \%$ BSA. Cells were incubated with fluorescent tagged secondary antibodies in 3\% BSA for $1 \mathrm{~h}$ at room temperature and the nuclei were counterstained with $4{ }^{\prime}$-6-diamidino-2-phenylindole (DAPI, $10 \mathrm{ng} / \mathrm{ml}$ ). Mounted slides were examined and images were acquired using a Zeiss LSM 510 Meta confocal microscope using 25X or $63 \mathrm{X}$ oil immersion lens. For the quantification of immunofluorescence signal, more than 50 cells were quantified using ImageJ software from three biological replicates and represented as mean $\pm \mathrm{SD}$. $P$ values were calculated by ANOVA method $\left({ }^{*} P \leq 0.05,{ }^{* *} P \leq 0.01\right.$, and $\left.{ }^{* * *} P \leq 0.001\right)$.

\section{Immunohistochemistry and analysis of patient samples}

Immunohistochemistry was performed using rectal adenocarcinoma tissue microarrays. Paraffin-embedded sections $(2 \mu \mathrm{m})$ were incubated in $100 \%$ xylene for $20 \mathrm{~min}$, followed by rehydration in descending dilutions of EtOH series (100\%, 90\%, and 70\%) before washing with $1 \mathrm{X}$ PBS. Slides were an antigen retrieval buffer (citrate buffer $\mathrm{pH} 6,0.05 \%$ Tween 20 ) for $3 \mathrm{~min}$ at $100{ }^{\circ} \mathrm{C}$. Tissue sections were allowed to cool to room temperature and washed with PBS three times, quenched for endogenous peroxidase activity with $3 \%$ hydrogen peroxide $\left(\mathrm{H}_{2} \mathrm{O}_{2}\right)$ treatment for $10 \mathrm{~min}$ at $\mathrm{RT}$, and then washed three times with PBS. Afterward, sections were blocked using SEA BLOCK blocking buffer for $20 \mathrm{~min}$ at RT followed by the primary antibody for H3K79me3 (1:250) overnight at $4{ }^{\circ} \mathrm{C}$. Sections were washed three times using PBS before adding the biotinylated secondary antibody (1:200, Envision Goat-anti-rabbit, Dako, Hamburg) and incubating for $1 \mathrm{~h}$ at RT. Sections were washed three times with PBS followed by Avidin-Peroxidase incubation diluted 1:1000 for $45 \mathrm{~min}$. Signals were detected using diaminobenzidine (DAB) substrate (Dako, Hamburg) for $8 \mathrm{~min}$ at RT. Slides were washed and hematoxylin (Mayer's hemalaun solution) was used for counterstaining for $5 \mathrm{~min}$. Histological slides were imaged using an Axioscop microscope and ZEN software (Carl Zeiss, Jena, Germany). Publically available gene expression data from The Cancer Genome Atlas consortium were evaluated for DOT1L expression using the Protein Atlas (https://www.proteinatlas.org/) tool. Patients displaying "high" or "low" levels of DOT1L 
expression were defined using the cutoff of 4.7 reads per million per kilobase (RPKM).

\section{SDS-PAGE and Western blot analyses}

Total cell protein lysates were prepared by lysing cells in RIPA (radio immunoprecipitation buffer) containing $1 \mathrm{X}$ PBS, 1\% NP-40, 0.5\% sodium deoxycholate, 0.1\% SDS, $1 \mathrm{mM}$ Pefabloc, $1 \mathrm{ng} / \mu \mathrm{L}$ Aprotinin/Leupeptin, $10 \mathrm{mM}$ $\beta$-glycerophosphate, and $1 \mathrm{mM} N$-ethylmaleimide. The lysates were resolved by SDS-PAGE and blotted to nitrocellulose membranes and then blocked with either 3\% BSA or $5 \%$ non-fat milk. The membranes were incubated with primary antibodies overnight at $4{ }^{\circ} \mathrm{C}$ followed by incubation with secondary antibodies coupled to horseradish peroxidase at RT. The membranes were developed using enhanced chemiluminescence and images were obtained using a ChemiDoc ${ }^{\mathrm{Tm}}$ XRS + System (Bio-Rad, California, USA). The list of antibodies used in this study is listed in Table 3.

\section{Analysis of DSB repair by GFP-based reporter assay}

To measure the repair efficiency of DNA double-strand breaks, DSB reporter assays were used as described [34]. To measure the HR and NHEJ efficiency, HeLa cells containing the stably integrated reporter construct pGC for $\mathrm{HR}$ and $\mathrm{pEJ}$ for NHEJ were transfected with DOT1L siRNA 1 or 4 . After $48 \mathrm{~h}$ of transfection, cell were transfected with the I-SceI endonuclease expression vector (pCMV3xnls-I-SceI, a kind gift from M. Jasin) to induce DSBs and pCMV-Neo as a control vector. After $24 \mathrm{~h}$ of DSB induction, cells were analyzed by flow cytometry
(FACScan, BD Bioscience) for GFP-positive cells as an indication of $\mathrm{HR}$ and NHEJ efficiency. Data were normalized to transfection efficiency and represented as mean $\pm \mathrm{SD}(n=3)$. For HR assay, HCT116 cells harboring stably integrated pHPRT-pDRGFP plasmid were transfected with the indicated siRNAs. After $24 \mathrm{~h}$ of siRNA transfections, cells were transfected with pCBASceI (Addgene) to induce DSBs and pEGFP as a positive control by electroporation. To analyze HR efficiency, GFP expression was quantified by flow cytometry using BD FACS Canto II (BD FACSDiva ${ }^{\mathrm{Tm}}$ 6.1.3, BD Biosciences) after $48 \mathrm{~h}$ of transfection. Additional information about flow cytometric analysis is provided in Additional file 4: supplementary methods.

\section{Cell proliferation assay}

For cell proliferation assay, approximately 1000-2000 cells were seeded in 96-well plates per well. After $24 \mathrm{~h}$, cells were treated with individual or combinations of compounds every $48 \mathrm{~h}$. Cell confluence was measured every 24 h using a Celigo Cytometer (Cyntellect Inc., USA). For colony formation assay with irradiation, cells were transfected with siRNA as mentioned above. After $48 \mathrm{~h}$ of transfection, cells were seeded (720-3000 cells/well) and irradiated with a single dose of 1, 2, 4, 6, and 8 Gy (Gulmay Medical, Camberley, UK), and a standard colony-forming assay was performed to determine the respective surviving fractions (SF). After 19 days, the colonies were fixed with $70 \%$ ethanol for $20 \mathrm{~min}$ and stained with Mayer's hemalaun for $5 \mathrm{~min}$ and colonies

Table 3 List of antibodies used in this study

\begin{tabular}{llll}
\hline Name & Cat. No & Company & Application \\
\hline YH2AX & 05-636, IF & Willipore & WB, IF \\
53BP1 & Sc-22760 & Santa Cruz & WB \\
ATM & A1106 & Sigma & WB \\
PATM (Ser1981) & 4526 & Cell Signaling & WB \\
CHIP & 61141 & Active Motif & WB \\
DOT1L & A310-953A & Bethyl Laboratories & WB \\
H2B & ab19847 & Abcam & WB \\
H3 & ab10799 & Abcam & WB, IF \\
H3K79me3 & C15410068 (pAb-068) & Diagenode & WB \\
H3K9me3 & & Diagenode & WB \\
H3K9me2 & mAb154-050 & Diagenode & WB \\
HSC70 & Sc-7298 & Santa Cruz & WB \\
KAP1 & A300-274A & Bethyl Laboratories & WB, IF \\
PKAP1 (S824) & A300-767A & Bethyl Laboratories & WB \\
RAD51 & Sc-8349 & Santa Cruz & WB \\
PRAD51 & ab111568 & Abcam & WB \\
RPA1 & NA13 & Calbiochem & \\
\hline
\end{tabular}


( $>50$ cells) were counted and SF $(n=3)$ were represented in the graph [35]. Data were normalized to plating efficiency for each condition and are represented as mean $(n=3) \pm$ SD. $P$ values were calculated by ANOVA method ( ${ }^{*} P \leq 0.05,{ }^{* *} P \leq 0.01$, and $\left.{ }^{* * *} P \leq 0.001\right)$.

\section{Chromatin fractionation}

Chromatin fractionation was performed as previously described [34]. Briefly, cells were re-suspended in lysis buffer [10 mM HEPES (pH 7.9), $10 \mathrm{mM} \mathrm{KCl,} 1.5 \mathrm{mM} \mathrm{MgCl}$, $0.34 \mathrm{M}$ sucrose, $10 \%$ glycerol, $0.1 \%$ Triton X-100, $1 \mathrm{mM}$ DTT, $1 \mathrm{mM}$ Pefabloc, $1 \mathrm{ng} / \mu \mathrm{L}$ Aprotinin/Leupeptin, $10 \mathrm{mM} \beta$-glycerophosphate, and $1 \mathrm{mM} \mathrm{N}$-ethylmaleimide] and nuclei were separated by centrifugation and then lysed in nuclear lysis buffer [3 mM EDTA, 0.2 mM EGTA, $1 \mathrm{mM}$ DTT, and protease inhibitors] for $30 \mathrm{~min}$ on ice. Soluble chromatin fractions were separated by centrifugation and analyzed by SDS-PAGE electrophoresis.

\section{Proximity ligation assay}

Proximity ligation assay (PLA) was performed using the Duolink $^{\circ}$ In Situ Red Starter Kit Mouse/Rabbit (DUO92101, Sigma-Aldrich, USA) according to the manufacturer's instructions. The cells were processed similar to immunofluorescence staining with specific primary antibodies. After that, cells were incubated with secondary antibodies conjugated to PLA probes followed by ligation and amplification according to the manufacturer's instructions. The images were acquired using a Zeiss LSM 510 Meta confocal microscope using 25X or $63 \mathrm{X}$ oil immersion lens.

\section{Statistical significance}

Experiments were performed in biological triplicates $(n=3)$ and the mean values are represented in the graph. The statistical significance (standard deviation, $\pm \mathrm{SD})$ was calculated and $p$ values were calculated using ANOVA represented as ns $-p>0.05, * p \leq 0.05$, *** $p \leq 0.01$, **** $p \leq 0.001$.

\section{Additional files}

Additional file 1: Figure S1. Knockdown of DOT1L leads to decreased H3K79 methylation. a SW837 cells were transfected with either control or individual or smart pool (4 siRNAs) DOT1L siRNAs (SP). After $72 \mathrm{~h}$ of transfection, relative DOT1L mRNA expression levels in control or DOT1L siRNA-transfected cells were measured using qPCR. b Similar to a, SW837 cells were transfected and total protein lysate was immunoblotted with DOT1L and H3K79me3 antibodies. H3 and HSC70 were used as loading controls. M, $\mathrm{G}$ and $\mathrm{N}$ were used as 3 independent controls ( $\mathrm{M}$ - mock, G - GL2 Duplex non-targeting siRNA, $N$ - siGENOME non-targeting siRNA). c Similar to Fig. 1b, U2OS cells were transfected with siDOT1L (smart pool) and treated with NCS for indicated time points and processed for immunofluorescence and stained with pKAP1 antibody. $d$ and e Quantification of Western blot data from Fig. 1d for represented proteins. f SW837 cells after the indicated time points following NCS $(100 \mathrm{ng} / \mathrm{ml})$ treatment. PLA assay was performed as described in Fig. If using $\mathrm{YH} 2 \mathrm{AX}$ and $53 \mathrm{BP} 1$ antibodies as a positive control and BSA as a negative control (scale bar - $10 \mu \mathrm{M}$ ). f Whole cell extracts from U2OS cells transfected similar to Fig. 1a were analyzed by Western blot for indicated proteins. (TIF $3056 \mathrm{~kb}$ )

Additional file 2: Figure S2. a HCT116 cells harboring a single copy of pHPRT-pDRGFP (HR substrate) were transfected with the indicated siRNAs and/or plasmid constructs as mentioned in the methods and proteins lysates were analyzed $48 \mathrm{~h}$ later by Western blot for the indicated proteins. b Whole cell extracts from U2OS cells were transfected similar to Fig. 1a and analyzed by Western blot for PRAD51. c Cell cycle analysis using SW837 cells transfected with either mock or DOT1L siRNA (smart pool) and after $48 \mathrm{~h}$ of transfection cells were treated with NCS for the indicated time points and processed for propidium iodide (PI) based flow cytometry as mentioned in the methods. The percentages of cells in each phase of cell cycle are represented in the graph $(n=3, \pm$ SD). (TIF $1026 \mathrm{~kb})$

Additional file 3: Figure S3. a SW837 cells were treated either with DMSO or the DOT1L inhibitor EPZ5676 $(1 \mu \mathrm{M})$ for either $3 \mathrm{~h}$ (acute) or 72 h (prolonged) followed by NCS $(100 \mathrm{ng} / \mathrm{ml})$ treatment for the indicated time points. Total protein lysates were analyzed by Western blot analysis for the indicated proteins. b Similar to a SW837 cells were treated either with DMSO or DOT1L inhibitor SGC094a (100 nM) followed by treatment with NCS. Total protein lysates were analyzed by Western blot for the indicated proteins. (TIF $572 \mathrm{~kb}$ )

Additional file 4: Supplementary Methods. (DOCX $25 \mathrm{~kb}$ )

\section{Abbreviations}

ATM: Ataxia telangiectasia mutated kinase; CRC: Colorectal cancer; CtIP: CtBPinteracting protein; PARP: Poly (adenosine diphosphate [ADP]) ribose polymerase; RPA: Replication protein A

\section{Acknowledgments}

The authors would like to thank N. Molitor for technical assistance and all members of the Johnsen group for helpful advice.

\section{Funding}

S.K.R. was supported by a "National Overseas Scholarship" (from the Ministry of Social Justice and Empowerment, Government of India).

\section{Authors' contributions}

SAJ and VK designed the study with input from FW, MS, HB, JG, and MG. JE performed IHC staining, JK scored tissue microarray data, and FK performed statistical analysis and graphical representation thereof. RLK analyzed TCGA gene expression data. VK, SKR, NÜ, WYM, and JH performed all cell culture experiments. All authors have read and approved the final version of the article, including the authorship list.

\section{Ethics approval and consent to participate}

The study was conducted in accordance with the Helsinki Declaration and was approved by the ethics committee of the University Medical Center Göttingen.

\section{Consent for publication}

All authors give consent for the publication of the manuscript.

\section{Competing interests}

The authors declare that they have no competing interests.

\section{Publisher's Note}

Springer Nature remains neutral with regard to jurisdictional claims in published maps and institutional affiliations.

\section{Author details}

${ }^{1}$ Department of General, Visceral and Pediatric Surgery, University Medical Center Göttingen, 37075 Göttingen, Germany. ${ }^{2}$ Department of Biotechnology, Rama Devi Women's University, Bhubaneswar 751022, India. ${ }^{3}$ Department of Pathology, University Medical Center Göttingen, 37075 Göttingen, Germany. ${ }^{4}$ Department of Medical Statistics, University Medical Center Göttingen, 37075 Göttingen, Germany. ${ }^{5}$ Department of Computer Science, University Augsburg, 86159 Augsburg, Germany. ${ }^{6}$ Institute of Molecular Oncology, Section for Cellular Oncology, Göttingen Center for Molecular Biosciences (GZMB) and University Medical Center, University of 
Göttingen, 37077 Göttingen, Germany. ${ }^{7}$ Laboratory of Radiobiology and Experimental Radiooncology, University Medical Center Hamburg-Eppendorf, 20246 Hamburg, Germany. ${ }^{8}$ Tumor Biology Department, National Cancer Institute, Cairo University, Cairo 11796, Egypt.

\section{Received: 28 August 2018 Accepted: 18 December 2018}

Published online: 07 January 2019

\section{References}

1. Siegel RL, Miller KD, Jemal A. Cancer statistics, 2016. CA Cancer J Clin. 2016; 66:7-30.

2. Berg M, Genetic SK. Epigenetic traits as biomarkers in colorectal cancer. Int J Mol Sci. 2011:12:9426-39.

3. Guinney J, Dienstmann R, Wang X, de Reyniès A, Schlicker A, Soneson C, et al. The consensus molecular subtypes of colorectal cancer. Nat Med. 2015; 21:1350-6.

4. Markowitz SD, Bertagnolli MM. Molecular origins of cancer: molecular basis of colorectal cancer. N Engl J Med. 2009;361:2449-60.

5. Aziz MA, Yousef Z, Saleh AM, Mohammad S, Al Knawy B. Towards personalized medicine of colorectal cancer. Crit Rev Oncol Hematol. 2017; 118:70-8.

6. Negrini S, Gorgoulis VG, Halazonetis TD. Genomic instability-an evolving hallmark of cancer. Nat Rev Mol Cell Biol. 2010;11:220-8.

7. Price BD, D'Andrea AD. Chromatin remodeling at DNA double-Strand breaks. Cell. 2013;152:1344-54.

8. Singer MS, Kahana A, Wolf AJ, Meisinger LL, Peterson SE, Goggin C, et al. Identification of high-copy disruptors of telomeric silencing in Saccharomyces cerevisiae. Genetics. 1998;150:613-32.

9. Nguyen AT, Zhang Y. The diverse functions of Dot1 and H3K79 methylation. Genes Dev. 2011;25:1345-58.

10. Zhu B, Chen S, Wang H, Yin C, Han C, Peng C, et al. The protective role of DOT1L in UV-induced melanomagenesis. Nat Commun. 2018;9:259.

11. McLean CM, Karemaker ID, van Leeuwen F. The emerging roles of DOT1L in leukemia and normal development. Leukemia 2014;28:2131-2138.

12. Oksenych V, Zhovmer A, Ziani S, Mari P-O, Eberova J, Nardo T, et al. Histone methyltransferase DOT1L drives recovery of gene expression after a genotoxic attack. PLoS Genet. 2013:9:e1003611.

13. Wakeman TP, Wang Q, Feng J, Wang X-F. Bat3 facilitates H3K79 dimethylation by DOT1L and promotes DNA damage-induced 53BP1 foci at G1/G2 cell-cycle phases. EMBO J. 2012;31:2169-81.

14. Li T, Liu Q, Garza N, Kornblau S, Jin VX. Integrative analysis reveals functional and regulatory roles of $\mathrm{H} 3 \mathrm{~K} 79 \mathrm{me} 2$ in mediating alternative splicing. Genome Med. 2018;10:30.

15. Jones B, Su H, Bhat A, Lei H, Bajko J, Hevi S, et al. The histone H3K79 methyltransferase Dot1L is essential for mammalian development and heterochromatin structure. PLoS Genet. 2008:4:e1000190.

16. Bernt KM, Zhu N, Sinha AU, Vempati S, Faber J, Krivtsov AV, et al. MLLrearranged leukemia is dependent on aberrant H3K79 methylation by DOT1L. Cancer Cell. 2011;20:66-78.

17. Chang M-J, Wu H, Achille NJ, Reisenauer MR, Chou C-W, Zeleznik-Le NJ, et al. Histone H3 lysine 79 methyltransferase Dot1 is required for immortalization by MLL oncogenes. Cancer Res. 2010;70:10234-42.

18. Krivtsov AV, Feng Z, Lemieux ME, Faber J, Vempati S, Sinha AU, et al. H3K79 methylation profiles define murine and human MLL-AF4 leukemias. Cancer Cell. 2008:14:355-68.

19. Daigle SR, Olhava EJ, Therkelsen CA, Majer CR, Sneeringer CJ, Song J, et al. Selective killing of mixed lineage leukemia cells by a potent small-molecule DOT1L inhibitor. Cancer Cell. 2011;20:53-65.

20. Daigle SR, Olhava EJ, Therkelsen CA, Basavapathruni A, Jin L, Boriack-Sjodin PA, et al. Potent inhibition of DOT1L as treatment of MLL-fusion leukemia. Blood. 2013;122:1017-25.

21. Liu W, Deng L, Song Y, Redell M. DOT1L inhibition sensitizes MLLrearranged AML to chemotherapy. PLoS One. 2014;9:e98270.

22. Gaedcke J, Leha A, Claus R, Weichenhan D, Jung K, Kitz J, et al. Identification of a DNA methylation signature to predict disease-free survival in locally advanced rectal cancer. Oncotarget. 2014:5:8123-35.

23. Bryant HE, Schultz N, Thomas HD, Parker KM, Flower D, Lopez E, et al. Specific killing of BRCA2-deficient tumours with inhibitors of poly (ADPribose) polymerase. Nature. 2005;434:913-7.

24. Helleday $T$, Petermann $E_{1}$ Lundin $C$, Hodgson B, Sharma RA. DNA repair pathways as targets for cancer therapy. Nat Rev Cancer. 2008;8:193-204.
25. Helleday T, Lo J, van Gent DC, Engelward BP. DNA double-strand break repair: from mechanistic understanding to cancer treatment. DNA Repair. 2007;6:923-35

26. Sartori AA, Lukas C, Coates J, Mistrik M, Fu S, Bartek J, et al. Human CtIP promotes DNA end resection. Nature. 2007;450:509-14.

27. Song Y, Li L, Chen Y, Liu J, Xiao S, Lian F, et al. Discovery of potent DOT1L inhibitors by AlphaLISA based high throughput screening assay. Bioorg Med Chem. 2018;26:1751-8.

28. Stein EM, Garcia-Manero G, Rizzieri DA, Tibes R, Berdeja JG, Savona MR, et al. The DOT1L inhibitor pinometostat reduces H3K79 methylation and has modest clinical activity in adult acute leukemia. Blood. 2018:131:2661-9.

29. Douillard JY, Cunningham D, Roth AD, Navarro M, James RD, Karasek P, et al. Irinotecan combined with fluorouracil compared with fluorouracil alone as first-line treatment for metastatic colorectal cancer: a multicentre randomised trial. Lancet Lond Engl. 2000;355:1041-7.

30. Saltz LB, Cox JV, Blanke C, Rosen LS, Fehrenbacher L, Moore MJ, et al. Irinotecan plus fluorouracil and leucovorin for metastatic colorectal cancer. Irinotecan Study Group. N Engl J Med. 2000;343:905-14.

31. Dietlein F, Thelen L, Reinhardt HC. Cancer-specific defects in DNA repair pathways as targets for personalized therapeutic approaches. Trends Genet. 2014;30:326-39.

32. Goodarzi AA, Noon AT, Deckbar D, Ziv Y, Shiloh Y, Löbrich $M$, et al. ATM signaling facilitates repair of DNA double-strand breaks associated with heterochromatin. Mol Cell. 2008:31:167-77.

33. White D, Rafalska-Metcalf IU, Ivanov AV, Corsinotti A, Peng H, Lee S-C, et al. The ATM substrate KAP1 controls DNA repair in heterochromatin: regulation by HP1 proteins and serine 473/824 phosphorylation. Mol Cancer Res. 2012; 10:401-14.

34. Kari V, Mansour WY, Raul SK, Baumgart SJ, Mund A, Grade M, et al. Loss of CHD1 causes DNA repair defects and enhances prostate cancer therapeutic responsiveness. EMBO Rep. 2016:17(11):1609-23.

35. Spitzner M, Roesler B, Bielfeld C, Emons G, Gaedcke J, Wolff HA, et al. STAT3 inhibition sensitizes colorectal cancer to chemoradiotherapy in vitro and in vivo. Int J Cancer. 2013;134:997-1007.

36. Karpiuk O, Najafova Z, Kramer F, Hennion M, Galonska C, König A, et al. The histone $\mathrm{H} 2 \mathrm{~B}$ monoubiquitination regulatory pathway is required for differentiation of multipotent stem cells. Mol Cell. 2012;46:705-13.

Ready to submit your research? Choose BMC and benefit from:

- fast, convenient online submission

- thorough peer review by experienced researchers in your field

- rapid publication on acceptance

- support for research data, including large and complex data types

- gold Open Access which fosters wider collaboration and increased citations

- maximum visibility for your research: over $100 \mathrm{M}$ website views per year

At $\mathrm{BMC}$, research is always in progress.

Learn more biomedcentral.com/submissions 